\title{
Delirium and agitation at the end of life
}

\author{
Christian M G Hosker consultant liaison psychiatrist and lead clinician ${ }^{1}$, Michael I Bennett St Gemma's \\ professor of palliative medicine ${ }^{2}$
}

${ }^{1}$ Leeds Liaison Psychiatry Service, Leeds and York Partnership Foundation Trust, Leeds LS9 7BE, UK; ${ }^{2}$ Academic Unit of Palliative Care, Leeds Institute of Health Sciences, School of Medicine, University of Leeds, Leeds LS2 9LJ, UK

Delirium is common in the last weeks or days of life. ${ }^{1}$ It can be distressing for patients and those around them. Successful management involves excluding reversible causes of delirium and balancing drugs that may provoke or maintain delirium while appreciating that most patients want to retain clear cognition at the end of life.

\section{What is delirium?}

Delirium is the abrupt onset of fluctuating confusion, inattention, and reduced awareness of the environment. Symptoms can affect different areas of cognition (memory, orientation, language, visuospatial ability, or perception) and may include hallucinations and disturbances in the sleep-wake cycle (box 1). ${ }^{2}$ Delirium can therefore be distressing to people who experience it and those around them. ${ }^{3}$ Delirium is classified into hyperactive (restlessness and agitated behaviour predominate), hypoactive (drowsiness and inactivity predominate), and mixed subtypes. The more subtle changes associated with the hypoactive form are often missed. ${ }^{4}$

\section{What are the causes of delirium?}

Delirium occurs when physical factors, often occurring simultaneously (box 2), act on a physiologically vulnerable brain, leading to confusion, changes in perceptions, and altered behaviours. ${ }^{6}$ In the last days of life, delirium has been described as the visible culmination of end stage multiorgan failure compounded by other non-reversible factors. ${ }^{7}$

In an observational study of 213 terminally ill delirious patients with cancer, ${ }^{8}$ the main causes identified were hepatic failure, drugs, pre-renal azotemia, hyperosmolality, hypoxia, disseminated intravascular coagulation, organic damage to the central nervous system, infection, and hypercalcaemia. In the same study, the occurrence of hyperactive delirium and the requirement for sedation correlated with hepatic failure, opioids, and steroids, while dehydration related conditions were statistically significantly associated with hypoactive delirium.

\section{Who gets delirium at the end of life?}

A systematic review suggested that $13-42 \%$ of patients admitted to palliative care inpatient units have delirium. ${ }^{9}$ A cohort study of patients with advanced cancer on a palliative care inpatient unit estimated that this rose to $88 \%$ in the days and hours before death. ${ }^{1}$

Pre-existing cognitive impairment, increased age, an already severe illness state, and infection reduce the brain's ability to resist delirium. ${ }^{10}$ Smaller observational studies in cancer patients found that lower performance status, the presence of lung cancer, and shorter time since diagnosis were associated with developing cognitive impairment. ${ }^{11}$ A retrospective study in cancer patients reported that male sex and the presence of a primary or metastatic brain tumour predisposed to delirium. ${ }^{12}$

Poor prognostic factors for delirium, predictive of non-recovery from the episode, include severe delirium, irreversible precipitating factors, greater degree of cognitive impairment, the hypoactive subtype, and a previous episode of delirium. Delirium caused by hypoxic or metabolic encephalopathy is particularly resistant to reversal, ${ }^{7}$ whereas delirium precipitated by drugs, electrolyte abnormalities, and infection are more likely to be reversible. ${ }^{813}$

\section{How to identify delirium at the end of life}

The core clinical features of delirium are the rapid onset of disturbed attention, fluctuating levels of consciousness, cognitive impairment, psychomotor disturbance, and disruption of the sleep-wake cycle. In our experience, within palliative care these core features often show as purposeless repetitive movements, such as plucking at bed sheets and removing clothes, accompanied by moaning and facial grimacing. Emotional changes, such as fear, anxiety, and agitation are common. Multifocal myoclonus, which may reflect opioid toxicity, renal failure, or other drug related toxicity, may be seen. ${ }^{14}$ 


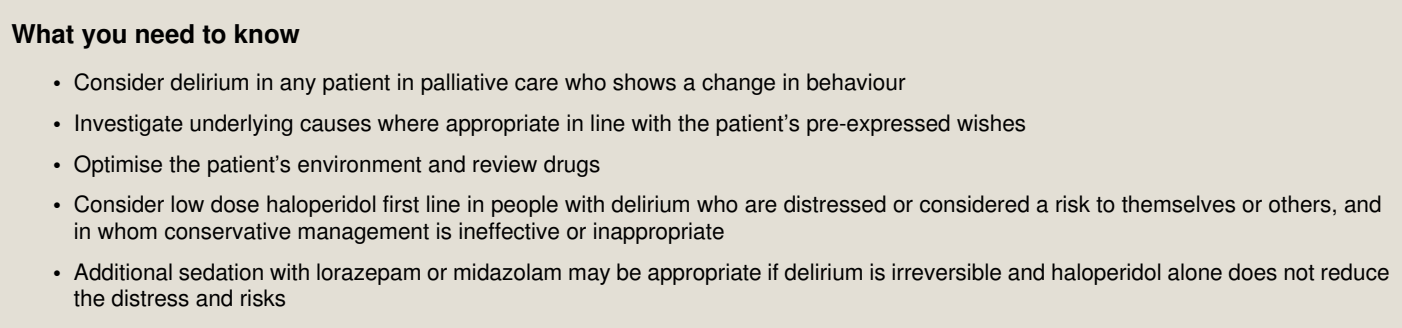

\title{
Sources and selection criteria
}

We searched Medline, Clinical Evidence, and the Cochrane Library using the terms delirium, terminal, terminal care, palliative care, palliative medicine, and end of life. Where possible we used systematic reviews and referenced these rather than the individual trials. Our search was limited to citations from 1990 to December 2015. We also searched the National Institute for Health and Care Excellence and the Scottish Intercollegiate Guidelines Network.

\author{
Box 1: What are the symptoms? \\ - Reduced level of consciousness \\ - Inability to focus and maintain attention \\ - Confusion and disorientation \\ - Daytime somnolence and restlessness at night \\ - Illusions and hallucinations \\ - Shifts from hypoactivity to hyperactivity \\ - Increased or decreased flow of speech \\ - Increased reaction time \\ - Enhanced startle reaction
}

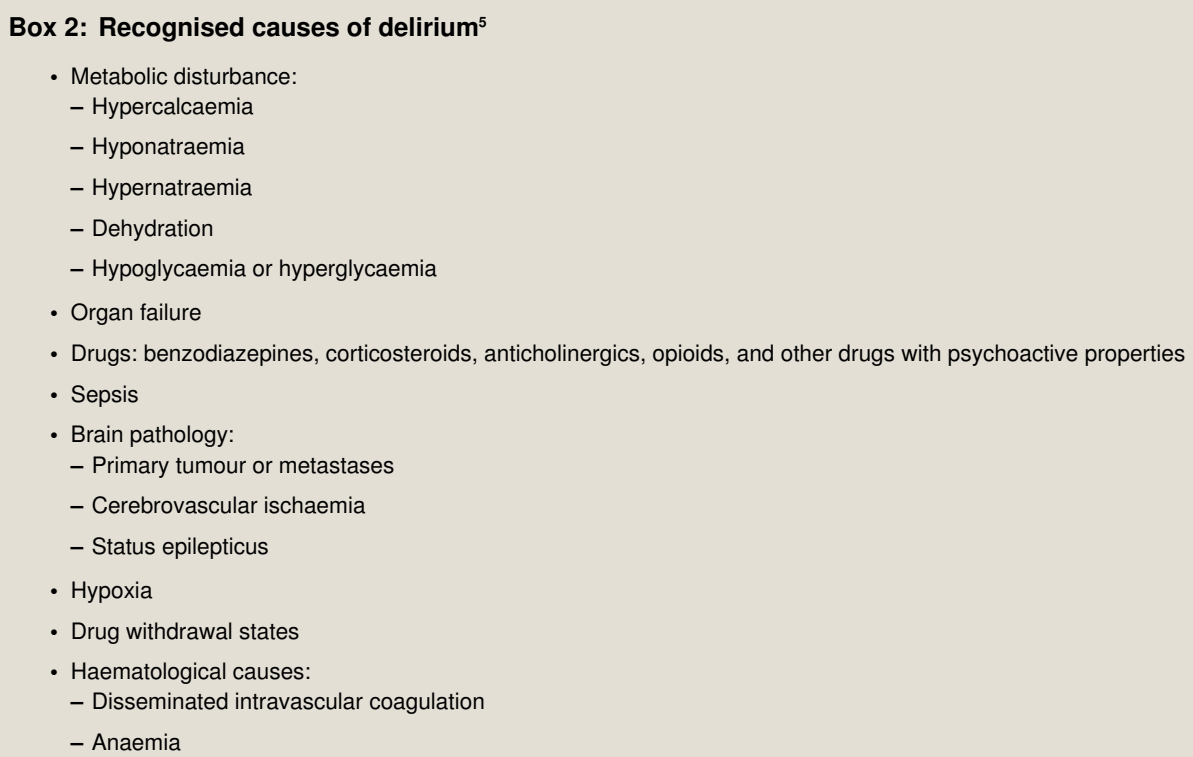

Validated tools exist for the screening and diagnosis of delirium (box 3 ) and can be used by non-specialists as a first step towards diagnosis. It is often not practical to use these tools in severe cases, where cooperation and communication are limited, and they often lack sufficient sensitivity and specificity to be solely relied on. Clinicians often use a global clinical assessment based on detailed history taking, information from care givers, and pattern recognition ${ }^{7}$ to make the diagnosis. If the diagnosis is in doubt, colleagues from palliative medicine or psychiatry can provide support.

\section{What investigations are required in palliative care?}

Delirium is often a progressive, irreversible process in the last stages of life. However, some causes, particularly drugs, may be reversible and probably account for $30-50 \%$ of cases. ${ }^{13}$ Decisions on whether to investigate should consider the likely nature of the underlying cause, the stage of the illness, and the goals of care pre-established with the patient (box 4). 


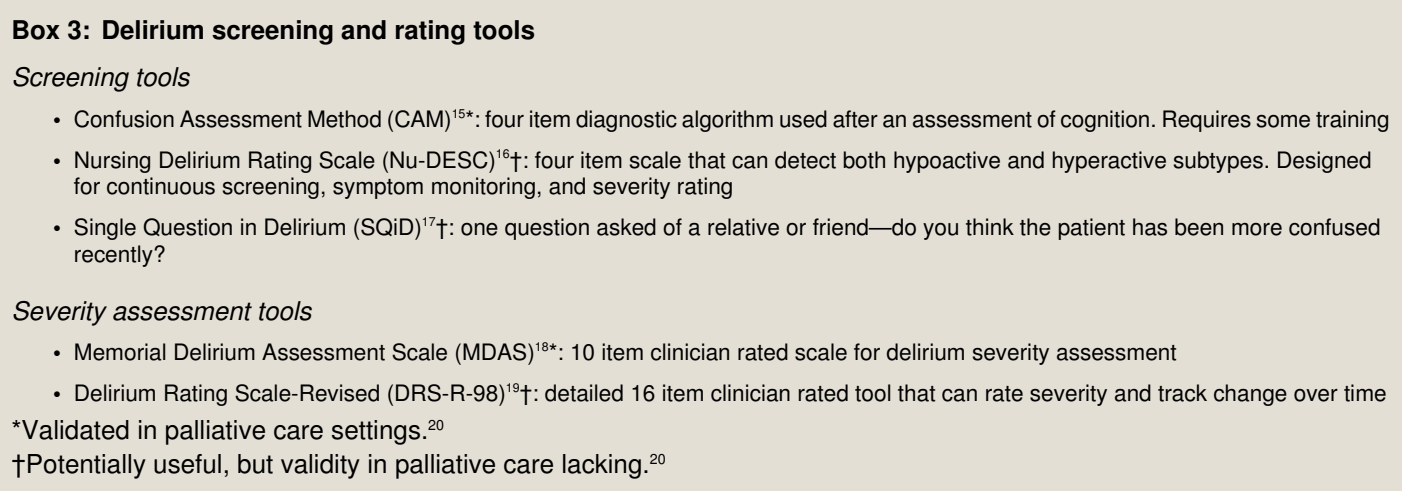

\section{Box 4: When and how to investigate}

- Consider the stage in the dying process and any agreed goals of care

- Review prescriptions for culpable drugs and the possibility of a withdrawal reaction

- Consider the possibility of alcohol or other substance withdrawal

- Use collateral history to determine rapidity of onset and baseline cognitive function

- If consistent with the goals of care, investigate for other underlying causes and treat where appropriate. Tests include:

- Investigations for metabolic and haematological abnormalities

- Blood cultures

- Midstream specimen of urine

- Chest radiography

- Lumbar puncture

- Electroencephalography

- Computed tomography or magnetic resonance imaging

\section{How is delirium managed in palliative care?}

There is robust evidence that multicomponent delirium prevention interventions, such as educating nursing staff, assessing and changing drugs, encouraging mobilisation, and improving the patient's environment, can reduce the incidence of delirium by about $30 \%$ in high risk medical and surgical inpatients, ${ }^{21}$ but evidence to support this approach in palliative care is lacking. ${ }^{22}$ Management of delirium in palliative care differs little from other settings in broad terms. The focus is on providing a safe optimised environment (box 5), treating reversible causes, controlling symptoms with drug and non-drug interventions, and communicating with patients and family members.

\section{Treating reversible causes}

Review drugs and modify those that cause or potentiate delirium (see box 2).

Overall, only about half of delirium episodes are potentially reversible, although the odds of reversibility are higher when opioids and other psychoactive drugs are precipitants. ${ }^{1}$ Because there are often several precipitants or predisposing factors, they all need to be dealt with simultaneously to reverse up to $50 \%$ of delirium episodes.

If there are concerns about neurotoxicity in patients taking opioids, consider a dose reduction or opioid switch. One observational study of 190 patients treated with opioids showed that 29 of 42 patients who developed confusion and 10 of 15 who developed hallucinations improved on opioid switching. ${ }^{23}$ A critical review in 2006 of opioid switching to reduce delirium suggested overall benefit, although the exact benefit was hard to define. ${ }^{24}$
A recent review of parenteral nutrition and hydration at the end of life suggested that delirium was the only aspect of terminal symptom control that was likely to benefit from hydration. ${ }^{25}$ If an infective cause is suspected, a trial of antibiotics may improve symptoms of delirium.

\section{Symptom control}

Medical strategies for delirium focus on reducing agitation and perceptual abnormalities.

Although there are several relevant clinical practice guidelines, ${ }^{10-28}$ a Cochrane review in $2012^{22}$ could not make any drug recommendations in end of life care because of the paucity of evidence. End of life care was specifically excluded from the National Institute for Health and Care Excellence (NICE) guidance on delirium, ${ }^{10}$ leaving clinicians without pragmatic guidance although, in practice, clinical management is broadly similar.

NICE guidance on delirium recommends considering short term haloperidol or olanzapine in people with delirium who are distressed or considered a risk to themselves or others and in whom verbal and non-verbal de-escalation techniques are ineffective or inappropriate. ${ }^{10}$ Although this guidance was not aimed at palliative care, it can help determine when antipsychotics are appropriate in delirium in palliative care. Haloperidol remains the drug of choice in delirium, despite limited evidence of efficacy from randomised controlled trials (RCTs). ${ }^{29}$ Like other antipsychotics, it is associated with extrapyramidal side effects in people over 70 years. ${ }^{30}$ It is less sedating than atypical antipsychotics such as risperidone or olanzapine and is useful where less sedation is required, such as in hypoactive states. A small RCT in 30 patients with AIDS related delirium compared haloperidol, chlorpromazine, and lorazepam. ${ }^{31}$ Patients in the haloperidol and chlorpromazine 


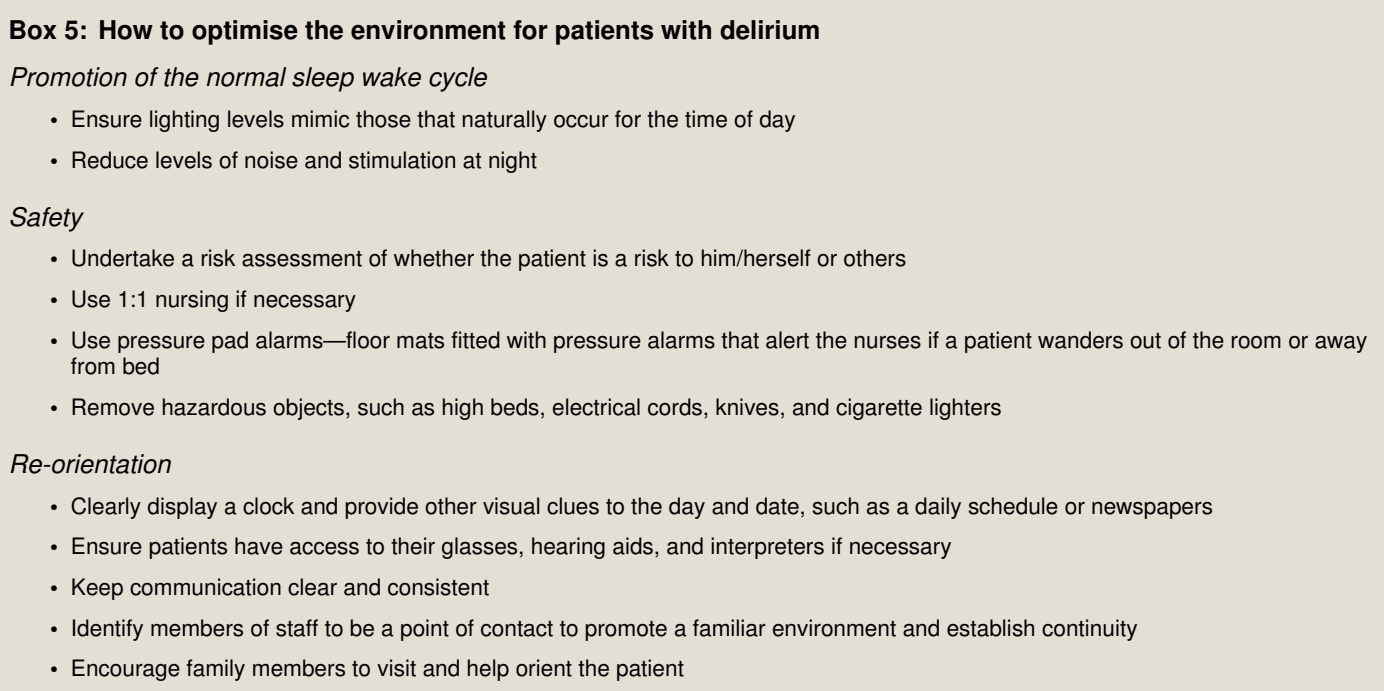

groups showed improvement in the delirium rating scale (DRS), whereas those who received lorazepam did not. Those taking lorazepam had greater side effects than those in the chlorpromazine and haloperidol groups. In an uncontrolled observational study of the harms and benefits of haloperidol in palliative care (119 inpatients and outpatients over 10 days), $12 \%$ of patients experienced side effects, most commonly somnolence or urinary retention. ${ }^{32}$

A recent RCT in 239 delirious palliative care patients (published abstract only) compared placebo, haloperidol, and risperidone over four days. ${ }^{33}$ Provisional results suggest patients prescribed haloperidol or risperidone had greater specific delirium symptoms (communication, behaviour, and perceptual disturbance) at study end than those prescribed placebo and also required more midazolam rescue doses.

The low doses of antipsychotics used for delirium are less likely to be associated with extrapyramidal side effects, but avoid combinations of antipsychotics and other dopamine blocking agents such as metoclopramide. Medium to longer term use of antipsychotics is also associated with increased risk of stroke, ${ }^{34}$ but these drugs are likely to be used short term in end of life care.

Despite NICE recommending antipsychotic options for delirium, ${ }^{10}$ benzodiazepines are preferred when Lewy body dementia or Parkinson's disease coexist because they do not exacerbate extrapyramidal symptoms. Patients with severe agitation (anxious, restless, and aggressive behaviour) may also require a short acting benzodiazepine, such as lorazepam or midazolam. This can be combined with haloperidol.

\section{Management of refractory delirium}

A recent systematic review suggested that refractory delirium is the most common reason for palliative sedation at the end of life. ${ }^{35}$ Palliative sedation is the monitored use of drugs to reduce awareness in dying patients and relieve suffering from refractory symptoms. The same systematic review found no evidence that the use of such proportionate sedation shortened life, although this finding was based on non-randomised samples.

Palliative sedation may be appropriate for patients with advanced disease at the end of life when the cause of delirium is judged to be irreversible; the manifestations of delirium, such as frightening cognitions and hallucinations, overwhelm the patient; or the patient's behaviour is a threat to him/herself or others. The aim is to control distress during the dying process. Sedation is a consequence rather than the objective of treatment. Sedating antipsychotics such as levomepromazine (methotrimeprazine) are titrated alone or together with a benzodiazepine such as midazolam. These are usually given as a subcutaneous infusion but can be given as regular injections during a titration period or when infusion devices are unavailable. In rare cases where these combinations do not control distress, phenobarbitone injections or infusions can be used, although these recommendations are not based on evidence from clinical trials and would require specialist palliative medicine expertise.

\section{Helping families understand delirium}

Delirium can be distressing for families. ${ }^{36}$ The hypoactive form is often missed by clinicians, and family members may be better placed to report the subtle changes in presentation that may herald its onset. A detailed observational study of the experience of delirium in inpatients with cancer found that mean scores for distress were higher in the spouses and family care givers who had witnessed the delirium than in affected patients. ${ }^{37}$

Furthermore, a qualitative study of 37 family care givers found that many thought that analgesics caused the delirium. ${ }^{36}$ The same study found that distress was lowest in care givers who were educated about the risk of delirium before it occurred.

Discuss delirium, the likely causes, and what it means for the patient with family care givers (box 6). Where poor prognostic factors are identified, discuss this with families so they are aware that their relative may be entering the last few days of life and that sedation may be needed for distressing or refractory symptoms.

Contributors: MIB conceived the idea for the review; $\mathrm{CMGH}$ and MIB wrote and approved the final version. MIB is guarantor.

Competing interests: We have read and understood BMJ policy on declaration of interests and declare the following interests: None. Provenance and peer review: Not commissioned; externally peer reviewed.

1 Lawlor PG, Gagnon B, Mancini IL, et al. Occurrence, causes, and outcome of delirium in patients with advanced cancer: a prospective study. Arch Intern Med 2000;160:786-94. doi:10.1001/archinte.160.6.786 pmid:10737278.

2 American Psychiatric Association. Diagnostic and statistical manual of mental disorders. (5th ed): DSM-5. 2013

3 Hosie A, Agar M, Lobb E, Davidson PM, Phillips J. Palliative care nurses' recognition and assessment of patients with delirium symptoms: a qualitative study using critical incident technique. Int J Nurs Stud 2014;51:1353-65. doi:10.1016/j.jnurstu.2014.02.005 pmid: 24630378. 


\section{Box 6: Communication with families and friends}

- Explain that the patient's concentration and memory are impaired, and that delirium probably reflects how frail the patient has become as a result of the illness and infection or the effect of drugs

- Suggest that limited investigations (such as blood tests) and a drugs review might help to diagnose and reverse the delirium if consistent with any advance care plan

- Highlight the importance of a calm and consistent environment

- Explain that this may be the start of a more rapid and irreversible decline in the patient's health

- Whether the delirium is reversible or not, provide reassurance that control of the patient's distress will be your priority

\section{Education into practice}

- Do you routinely observe and ask during consultations whether your medically ill patients are alert and orientated?

- Does your team know how to recognise and manage delirium that is distressing to patients or putting them or others at risk of harm?

\section{Questions for future research}

- What strategies are effective for the prevention of delirium in palliative care? ? $^{38}$

- How effective or harmful are antipsychotics compared with placebo, and at what doses?

- How effective or otherwise are non-pharmacological strategies?

- What are the most effective strategies for supporting families of those affected by delirium?

\section{Further sources of information for patients and professionals}

Apps

- Confusion: Delirium and Dementia Application. Available free from iTunes and Google Play. Funded by Health Education England. Guidance on assessing and managing patients across settings aimed at doctors in training. It includes interactive, validated assessment scales and management advice

- Delirium Learning Application. Available free from iTunes and Google Play. Developed by NHS Education for Scotland. Provides accessible advice on the assessment and immediate management of delirium

- Vancouver Island Healthcare (http://www.viha.ca/mhas/resources/delirium/tools.htm). Provides links to rating scales and information resources that can aid assessment and be used to educate patients

- European Delirium Association (http://www.europeandeliriumassociation.com).http://www.europeandeliriumassociation.com)./ Provides delirium information resources for health professionals and patients

- National Institute for Health and Care Excellence. (http://www.nice.org.uk/guidance/CG103/ifp/chapter/More-information). Provides general information for those directly affected by delirium and their carers. It is not aimed at those at the end of life, but most of the information is highly relevant

- Royal College of Psychiatrists (http://www.rcpsych.ac.uk/expertadvice/problems/physicalillness/delirium.aspx). Provides a brief and accessible information resource aimed at patients and carers

\section{How patients were involved in the creation of this article}

No patients were directly involved in the creation of this article

4 Fang C-K, Chen H-W, Liu S-I, Lin CJ, Tsai LY, Lai YL. Prevalence, detection and treatment of delirium in terminal cancer inpatients: a prospective survey. Jpn J Clin Oncol 2008;38:56-63. doi:10.1093/jico/hym155 pmid:18238881.

5 LeGrand SB. Delirium in palliative medicine: a review. J Pain Symptom Manage 2012;44:583-94. doi:10.1016/j.jpainsymman.2011.10.013 pmid:22682074.

6 Inouye SK, Charpentier PA. Precipitating factors for delirium in hospitalized elderly persons. Predictive model and interrelationship with baseline vulnerability. JAMA 1996;275:852-7. doi:10.1001/jama.1996.03530350034031 pmid:8596223.

7 Bush SH, Leonard MM, Agar M, et al. End-of-life delirium: issues regarding recognition, optimal management, and the role of sedation in the dying phase. J Pain Symptom Manage 2014:48:215-30. doi:10.1016/j.jpainsymman.2014.05.009 pmid:24879997.

8 Morita T, Tei Y, Tsunoda J, Inoue S, Chihara S. Underlying pathologies and their associations with clinical features in terminal delirium of cancer patients. J Pain Symptom Manage 2001;22:997-1006. doi:10.1016/S0885-3924(01)00360-8 pmid:11738162.

9 Hosie A, Davidson PM, Agar M, Sanderson CR, Phillips J. Delirium prevalence, incidence, and implications for screening in specialist palliative care inpatient settings: a systematic review. Palliat Med 2013:27:486-98. doi:10.1177/0269216312457214 pmid:22988044.

10 National Institute for Health and Care Excellence. Delirium: prevention, diagnosis and management. NICE guideline 103. 2010. https://www.nice.org.uk/guidance/CG103? UNLID=71857637201581742332.

11 Kurita GP, Sjøgren P, Ekholm O, et al. Prevalence and predictors of cognitive dysfunction in opioid-treated patients with cancer: a multinational study. J Clin Oncol2011;29:1297-303. doi:10.1200/JCO.2010.32.6884 pmid:21357785

12 Cobb JL, Glantz MJ, Nicholas PK, et al. Delirium in patients with cancer at the end of life. Cancer Pract 2000;8:172-7. doi:10.1046/j.1523-5394.2000.84006.x pmid:11898256.

13 Leonard M, Raju B, Conroy M, et al. Reversibility of delirium in terminally ill patients and predictors of mortality. Palliat Med 2008:22:848-54. doi:10.1177/0269216308094520 pmid: 18755829.
14 Bush SH, Kanji S, Pereira JL, et al. Treating an established episode of delirium in palliative care: expert opinion and review of the current evidence base with recommendations for future development. J Pain Symptom Manage 2014;48:231-48. doi:10.1016/j.jpainsymman. 2013.07.018 pmid:24480529.

15 Inouye SK, van Dyck CH, Alessi CA, Balkin S, Siegal AP, Horwitz RI. Clarifying confusion: the confusion assessment method. A new method for detection of delirium. Ann Intern Med 1990;113:941-8. doi:10.7326/0003-4819-113-12-941 pmid:2240918.

16 Gaudreau JD, Gagnon P, Harel F, Tremblay A, Roy MA. Fast, systematic, and continuous delirium assessment in hospitalized patients: the nursing delirium screening scale. J Pain Symptom Manage 2005;29:368-75. doi:10.1016/j.jpainsymman.2004.07.009 pmid: 15857740.

17 Sands MB, Dantoc BP, Hartshorn A, Ryan CJ, Lujic S. A single question in delirium (SQiD): testing its efficacy against psychiatrist interview, the confusion assessment method and the memorial delirium assessment scale. Palliat Med 2010;24:561-5. doi:10.1177/ 0269216310371556 pmid:20837733.

18 Breitbart W, Rosenfeld B, Roth A, Smith MJ, Cohen K, Passik S. The memorial delirium assessment scale. J Pain Symptom Manage 1997:13:128-37. doi:10.1016/S0885-3924( 96)00316-8 pmid:9114631.

19 Trzepacz PT, Mittal D, Torres R, Kanary K, Norton J, Jimerson N. Validation of the Delirium Rating Scale-revised-98: comparison with the delirium rating scale and the cognitive test for delirium. J Neuropsychiatry Clin Neurosci 2001;13:229-42. doi:10.1176/jnp.13.2 229 pmid: 11449030.

20 Leonard MM, Nekolaichuk C, Meagher DJ, et al. Practical assessment of delirium in palliative care. J Pain Symptom Manage 2014;48:176-90. doi:10.1016/j.jpainsymman. 2013.10.024 pmid:24766745.

21 Siddiqi N, Harrison JK, Clegg A, et al. Interventions for preventing delirium in hospitalised non-ICU patients. Cochrane Database Syst Rev 2016:3:CD005563 pmid·26967259. 
22 Candy B, Jackson KC, Jones L, Leurent B, Tookman A, King M. Drug therapy for delirium in terminally ill adult patients. Cochrane Database Syst Rev 2012;11:CD004770.pmid: 23152226.

23 de Stoutz ND, Bruera E, Suarez-Almazor M. Opioid rotation for toxicity reduction in terminal cancer patients. J Pain Symptom Manage 1995;10:378-84. doi:10.1016/0885-3924(95) 90924-C pmid:7673770.

24 Mercadante S, Bruera E. Opioid switching: a systematic and critical review. Cancer Treat Rev 2006;32:304-15. doi:10.1016/j.ctrv.2006.03.001 pmid:16624490.

25 Dev R, Dalal S, Bruera E. Is there a role for parenteral nutrition or hydration at the end of life? Curr Opin Support Palliat Care 2012;6:365-70. doi:10.1097/SPC. ob013e328356ab4a pmid:22801468.

26 National Cancer Institute. Delirium (PDQ)—health professional version. 2015. http://www. cancer.gov/about-cancer/treatment/side-effects/memory/delirium-hp-pdq-

27 Scotland NHS. Palliative care guidelines: delirium. 2014. http://www. palliativecareguidelines.scot.nhs.uk/guidelines/symptom-control/Delirium.aspx.

28 Canadian Coalition for Seniors' Mental Health. Guideline on the assessment and treatment of delirium in older adults at the end of life. 2010. http://www.ccsmh.ca/en/projects/delirium. cfm.

29 Seitz DP, Gill SS, van Zyl LT. Antipsychotics in the treatment of delirium: a systematic review. J Clin Psychiatry 2007:68:11-21. doi:10.4088/JCP.v68n0102 pmid:17284125.

30 Inouye SK, Marcantonio ER, Metzger ED. Doing damage in delirium: the hazards of antipsychotic treatment in elderly people. Lancet Psychiatry 2014;1:312-5. doi:10.1016/ S2215-0366(14)70263-9 pmid:25285270.

31 Breitbart W, Marotta R, Platt MM, et al. A double-blind trial of haloperidol, chlorpromazine, and lorazepam in the treatment of delirium in hospitalized AIDS patients. Am J Psychiatry 1996;153:231-7. doi:10.1176/ajp.153.2.231 pmid:8561204.
32 Crawford GB, Agar M M, Quinn SJ, et al. Pharmacovigilance in hospice/palliative care: net effect of haloperidol for delirium. J Palliat Med 2013:16:1335-41. doi:10.1089/jpm . 2013.0230 pmid:24138282

33 Agar M, Lawlor PG, Quinn SJ. Phase III randomized double-blind controlled trial of oral risperidone, haloperidol, or placebo with rescue subcutaneous midazolam for delirium management in palliative care. 2015 Annual American Geriatrics Society Meeting in Washington DC, USA, May 15th-17th 2015. J Am Geriatr Soc 2015;63(suppl 1):S99.

34 Douglas IJ, Smeeth L. Exposure to antipsychotics and risk of stroke: self controlled case series study. BMJ 2008;337:a1227. doi:10.1136/bmj.a1227 pmid:18755769.

35 Maltoni M, Scarpi E, Rosati M, et al. Palliative sedation in end-of-life care and survival: a systematic review. J Clin Oncol 2012;30:1378-83. doi:10.1200/JCO.2011.37.3795 pmid: 22412129.

36 Cohen MZ, Pace EA, Kaur G, Bruera E. Delirium in advanced cancer leading to distress in patients and family caregivers. J Palliat Care 2009;25:164-71.pmid:19824277.

37 Breitbart W, Gibson C, Tremblay A. The delirium experience: delirium recall and delirium-related distress in hospitalized patients with cancer, their spouses/caregivers, and their nurses. Psychosomatics 2002;43:183-94. doi:10.1176/appi.psy.43.3.183 pmid: 12075033.

38 Lawlor PG, Davis DH, Ansari M, et al. An analytical framework for delirium research in palliative care settings: integrated epidemiologic, clinician-researcher, and knowledge user perspectives. J Pain Symptom Manage 2014;48:159-75. doi:10.1016/j.jpainsymman. 2013.12.245 pmid:24726762.

Published by the BMJ Publishing Group Limited. For permission to use (where not already granted under a licence) please go to http://group.bmj.com/group/rights-licensing/ permissions 